\title{
COST AND PERFORMANCE OF RAPID-CYCLING PROTON SYNCHROTRONS*
}

\author{
Charles M. Ankenbrandt, FNAL, Batavia, IL 60510, USA and \\ Rolland P. Johnson, Illinois Institute of Technology, Chicago, IL 60616 USA
}

\begin{abstract}
Alternative rapid-cycling proton synchrotrons using resonant magnet power supply systems are examined as possible replacements for the Fermilab 8-GeV Booster Synchrotron. The baseline machine, with $16 \mathrm{GeV}$ maximum kinetic energy, would replace the Booster and also function as the first stage of a neutrino factory. The study described here uses the cost estimates of the baseline design to scale costs for alternate machine designs with the same functional requirements as the baseline machine. Considering different maximum energy, magnet aperture, and injection strategies, it is found that considerable construction cost savings can be achieved by means of lower energy designs with smaller circumference. The costs are dominated by the stored energy of the resonant magnet power supply system and the volts/turn of the rf accelerating system. Additional considerations of packing fractions, maximum magnetic fields, and ease of implementation are also discussed.
\end{abstract}

\section{INTRODUCTION}

A study recently carried out at Fermilab [1] produced a baseline design of a $16-\mathrm{GeV}$ rapid-cycling proton synchrotron intended to replace the Fermilab Booster. As part of that study, the effects of changes in some of the major parameters on the cost and performance of such a facility were examined. To compare the costs of various alternatives, simple scaling rules were incorporated into a spreadsheet and applied to the estimated costs of major subsystems of the baseline design. Three specific studies were reported in Appendix $\mathrm{B}$ of the design document [2]: capital cost as a function of the maximum kinetic energy $\mathrm{T}_{\max }$ and the maximum magnetic field $\mathrm{B}_{\max }$, respectively, and a comparison of operating costs.

For the sake of brevity, only the study of cost vs. $\mathrm{T}_{\max }$ is described in detail in the present paper. Machines of 8,12 , and $16 \mathrm{GeV}$ maximum kinetic energy with $40 \pi$ $\mathrm{mm}$-mr transverse acceptance are modeled to show how their costs compare to each other and to the $16 \mathrm{GeV} 60 \pi$ baseline proton driver (bPD). These energies are chosen because $8 \mathrm{GeV}$ is considered the lowest energy compatible with present Booster functionality, $16 \mathrm{GeV}$ allows a comparison between two different transverse acceptances, and $12 \mathrm{GeV}$, besides being midway between the other two, is the actual stage 1 energy of the bPD. The 8,12 , and $16 \mathrm{GeV}$ rings with $40 \pi$ acceptance have, respectively, circumferences of $0.75,1$, and 1.5 times that of the present Fermilab Booster.

\section{IMPORTANT PARAMETERS}

The parameters of the alternative proton drivers (PD) are varied in such a way as to produce performance equivalent to that of the baseline design. That is, for stage 1 Main Injector (MI) operation, at least $1.2 \times 10^{14}$ protons are delivered to the MI. For stage 2 Neutrino Factory operation, the beam power is $1 \mathrm{MW}$. In all cases, the parameters are adjusted to keep the calculated Laslett tune shift less than or equal to that of the bPD.

\subsection{Laslett space charge tune shift}

spread,

The Laslett incoherent space charge tune shift or

$$
\Delta v=\frac{3 \mathrm{f}_{\mathrm{T}} \mathrm{r}_{\mathrm{P}}}{2 \mathrm{~B}} \cdot \frac{\mathrm{N}_{\mathrm{P}}}{\beta \gamma^{2} \varepsilon_{\mathrm{N}}},
$$

is used as the touchstone in all the accelerator models discussed below and allows the parameters of the machines to be varied in a consistent manner. Here B and $\mathrm{f}_{\mathrm{T}}$, the bunching and transverse form factors, and $\Delta \mathrm{v}$ are held at the same values as in the bPD design. The number of protons $\left(\mathrm{N}_{\mathrm{P}}\right)$, the beam normalized transverse emittance $\left(\varepsilon_{\mathrm{N}}\right)$, and the injection energy (which determine the Lorentz parameters of $\beta$ and $\gamma$ ), are the variables used to equalize performance parameters of MI intensity (stage 1) and beam power (stage 2) for each design. The relativistic Lorentz factor $\beta \gamma$ for the $400 \mathrm{MeV}$ injection kinetic energy of the bPD is 1.02 , so that the geometrical acceptance $\varepsilon_{\mathrm{G}}$ is very nearly the same as the normalized emittance, $\varepsilon_{\mathrm{N}}=\beta \gamma \varepsilon_{\mathrm{G}}$.

\subsection{Circumference}

One parameter that does not appear explicitly in the above formula for the Laslett tune shift is the machine radius or circumference. Thus the maximum number of protons that can be stored in a ring limited by the Laslett tune shift is independent of its circumference. This important property can be used to advantage in the design of the 8 and $12 \mathrm{GeV}$ machines, which can have smaller circumferences than the $16 \mathrm{GeV}$ baseline machine. Reducing the circumference helps in a number of ways. Of course the civil construction cost is reduced. In addition, synchrotrons of smaller circumference will have a smaller transverse beam size and thus require less magnet aperture because each transverse beta function scales as the square root of the circumference. A smaller circumference also means that more batches (PD beam acceleration cycles) can be used to load the MI, so each batch can have fewer protons, allowing the transverse aperture to be reduced further while maintaining the same 
Laslett tune shift. This aspect is discussed further in section 2.4 on apertures.

Fewer rf cavities are needed as the circumference is reduced, since the beam passes through the cavities more often. Fewer cavities are needed, as well, if the machine energy is reduced so that the maximum $\mathrm{dE} / \mathrm{dt}$ is lowered. Approximately, then, a ring of half the energy and half the circumference will require only a quarter as many rf cavities. The bPD design assumes that the present Booster $53 \mathrm{MHz}$ rf cavities will be upgraded and used to provide acceleration in stage 1 to deliver beam to MI. While the reuse of these cavities does reduce stage 1 costs, it also limits the $16 \mathrm{GeV}$ ring to operation at 12 $\mathrm{GeV}$ and precludes simultaneous operation of the PD and Booster.

Since the fraction of the circumference occupied by rf cavities in a smaller, lower-energy machine is also reduced, the fraction of the ring used for other things can be increased. For example, a larger packing fraction (total bending magnet length/circumference), or more complex lattice design is easier to accomplish. As detailed in reference [1], the available space can be used to reduce costs by reducing $\mathrm{B}_{\max }$, thereby lowering the stored energy in the magnet and power supply systems. A lower $\mathrm{B}_{\max }$ also means that magnet saturation and induced dipole and quadrupole tracking problems are reduced.

\subsection{Injection Energy and Intensity}

For the alternative proton drivers described here, the choice has been made to rely on upgrading the existing $\mathrm{H}^{-}$source and the Linac to provide more protons for stage 2 beam power and/or to provide more energy to reduce the Laslett tune shift at injection by increasing $\beta^{2} \gamma^{3}$. This choice has the virtue of lowering costs for the proton driver itself by reducing both the required beam energy and magnet apertures. However, the costs for the Linac and source improvements, unlike the costs for the ring components, cannot be scaled from the baseline design.

Replacement of the Cockroft-Walton preaccelerators by RFQs, modifications to the initial drift tube structures of the $200 \mathrm{MHz}$ Linac, and improved $\mathrm{H}^{-}$ sources, which are part of the bPD project, are also included in each model in this paper at a cost of $\$ 5.5 \mathrm{M}$. Cost estimates for additional Linac and source improvements needed for some models have been generated by extrapolating from past experience. The Fermilab Linac energy upgrade done in 1992 cost about $\$ 2 \mathrm{M}$ for each $40 \mathrm{MeV}$ module. In the studies below we have assumed an inflation-adjusted cost of $\$ 2.67 \mathrm{M}$ per 40 $\mathrm{MeV}$. A combination of pulse length and beam current improvements is needed for the $\mathrm{H}^{-}$source to provide all that is required for the cases in the studies. Where more than $3 \times 10^{13}$ protons are needed from the Linac, a rather arbitrary figure of $\$ 2 \mathrm{M}$ for a source improvement program has been added. It is assumed that the source can be improved to provide the required number of protons within the present Linac pulse length of $\sim 100 \mu \mathrm{s}$ so that improvements to the Linac pulse forming networks will not be needed.

Raising the injection energy provides the capability to deliver brighter beam at the tune shift limit. An additional benefit from increased injection energy is higher injection velocity. This reduces the required frequency range of the rf system and perhaps, therefore, its cost and complexity. While $\Delta \mathrm{f} / \mathrm{f}=2\left(\mathrm{f}_{\text {ext }}-\mathrm{f}_{\text {inj }}\right) /\left(\mathrm{f}_{\text {ext }}+\right.$ $\left.\mathrm{f}_{\mathrm{inj}}\right)=33 \%$ for the bPD, it is only $\Delta \mathrm{f} / \mathrm{f}=13 \%$ for the 8 $\mathrm{GeV}$ model with $\mathrm{T}_{\text {Linac }}=0.73 \mathrm{GeV}$.

\subsection{Apertures}

The magnet good-field aperture usually determines the machine acceptance at the injection energy. In the $16 \mathrm{GeV}$ baseline Proton Driver design, the aperture is made very large (5 in. by 9 in.) to accommodate the design emittance $\varepsilon_{\mathrm{N}}$ of $60 \pi \mathrm{mm}-\mathrm{mr}$, $50 \%$ larger than the $40 \pi$ design acceptance of the MI at 8 $\mathrm{GeV}$. The bPD must have a large acceptance because its large circumference allows only 4 batches to be stacked in the MI. With only 4 batches, it is necessary to accelerate $3 \times 10^{13}$ protons per batch to reach the $1.2 \times 10^{14} \mathrm{MI}$ requirement. Thus the normalized emittance was increased from $40 \pi$ to $60 \pi$ to keep the Laslett tune shift fixed as the needed intensity increased from $2 \times 10^{13}$ to $3 \times 10^{13}$. Since the size of a $60 \pi$ beam at $12 \mathrm{GeV}$ is comparable to that of a $40 \pi$ beam at $8 \mathrm{GeV}$, there may be no problem with injection into the MI from the bPD. However that choice does require MI extraction and beam transport components to accommodate larger beam sizes.

By reducing the PD circumference to that of the present Booster $\left(\mathrm{C}_{\mathrm{B}}\right)$, one can inject 6 batches, each having $2 / 3$ the number of protons, into the MI, thereby providing the same total intensity with $\varepsilon_{\mathrm{N}}=40 \pi$ and the same Laslett tune shift as in the baseline PD. To reduce the circumference $\mathrm{T}_{\max }$ must also be lowered. Of course, fewer batches means that the MI can be loaded faster, which in the case of the 4-batch injection of the baseline PD implies a $7 \%$ increase in protons per hour for MI operation compared to the 6-batch injection from a ring of Booster circumference. On the other hand, a ring with circumference larger than that of the present Booster cannot efficiently create and store antiprotons in the Booster-sized Antiproton Collector. One third of the batch destined to hit the antiproton production target from the baseline PD should be without beam in this case, and if there were three other batches, this would lead to an $8 \%$ decrease in protons/hr from the MI.

To satisfy the stage 2 requirement of $1 \mathrm{MW}$ on target from a machine having lower top energy, it is necessary to accelerate more protons. Larger $\mathrm{N}_{\mathrm{P}}$ would increase the Laslett tune shift unless the normalized emittance and/or the injection energy are increased. The algorithm used in the spreadsheet studies is to increase the Linac energy enough that the larger $\beta^{2} \gamma^{3}$ makes up for the increase in protons.

A major advantage in using a smaller magnet aperture is the reduced cost for the magnet and power 
supply systems. The costs of magnets, chokes, and capacitors in the resonant system are proportional to the stored energy in the ring magnets,

$$
\text { Stored Energy }=\mathrm{k} \sum_{\text {magnets }} \mathrm{B}_{\max }{ }^{2} \mathrm{~L} \mathrm{~A}_{\mathrm{T}} \text {, }
$$

where $L$ is the effective length and $A_{T}$ is the area of the transverse aperture of each magnet. $\mathrm{A}_{\mathrm{T}}$ depends on a combination of the acceptance requirements for the needed beam emittance and the momentum acceptance. For injection, only a few $\mathrm{mm}$ of momentum acceptance are needed and the geometric aperture is primarily determined by $\varepsilon_{\mathrm{G}}$. At extraction energies, large momentum acceptance is needed to allow short bunches to be formed. In this case, the horizontal emittance is damped by the $\beta \gamma$ adiabatic damping factor so that when the momentum excursion is added, the total beam size fits within the acceptance determined by the injection requirements.

\section{COST AS A FUNCTION OF $T_{\text {max }}$}

The magnet physical apertures for the alternative proton drivers correspond to fixed geometric transverse acceptances of $40 \pi \mathrm{mm}-\mathrm{mr}$. A single value for all three energies simplifies the comparison of other variables. This acceptance corresponds to the design acceptance of the MI and seems adequate to satisfy the performance requirements. (Note that the $16 \mathrm{GeV}$ driver considered here is not the same as the baseline PD, which has an acceptance of $60 \pi$. Note also that the costs of the stage 1 baseline PD in the spreadsheet include only enough power supply to reach $12 \mathrm{GeV}$.) Another parameter held constant in this study is the maximum dipole magnetic field of $1.5 \mathrm{~T}$ used in the baseline design.

The variables used to satisfy the performance requirements for the three different energies are then the machine circumference and the $\mathrm{H}^{-}$source and Linac parameters (number of protons and injection energy). The circumference is chosen to maximize the number of batches to be injected into the MI while keeping the packing fraction reasonable. For the three energies of 8 , 12 , and $16 \mathrm{GeV}$, circumferences of $3 / 4,1$, and $1.5 \mathrm{C}_{\mathrm{B}}$, respectively, seem reasonable, where $C_{B}=474.2 \mathrm{~m}$ is the circumference of the present Fermilab Booster. Considerable source development will be needed to achieve the stage 2 Linac intensity parameters for the 8 $\mathrm{GeV}$ case, although stage 1 for that energy serves the MI well because of the larger number of batches that can be injected. Increasing the Linac energy seems rather straightforward, and space for this has been allocated in the bPD design.

The cost estimates of the baseline PD are used to scale costs and performance for these machines. The cost of a machine is assumed to be made up of things proportional to: 1) stored energy (magnets and power supplies, half of utilities), 2) rf volts per turn (cavities and their supplies), 3) tunnel length (conventional construction, vacuum system, half of utilities, project management) and 4) constants (Linac Front-end improvements). When required, increased Linac energy (taken to be $\sim \$ 2.7 \mathrm{M} / 40 \mathrm{MeV}$ module) and $\mathrm{H}^{-}$source development $(\$ 2 \mathrm{M})$ are also included.

The results of the calculation are shown in Figure 1. The spreadsheets can be seen in Appendix B of the Fermilab Proton Driver Study [2]. The costs increase faster than linearly with $\mathrm{T}_{\max }$, reflecting the rapidly rising costs of additional rf and magnet systems needed for higher energy and larger circumference.

\section{Figure 1. Construction Cost vs Tmax}

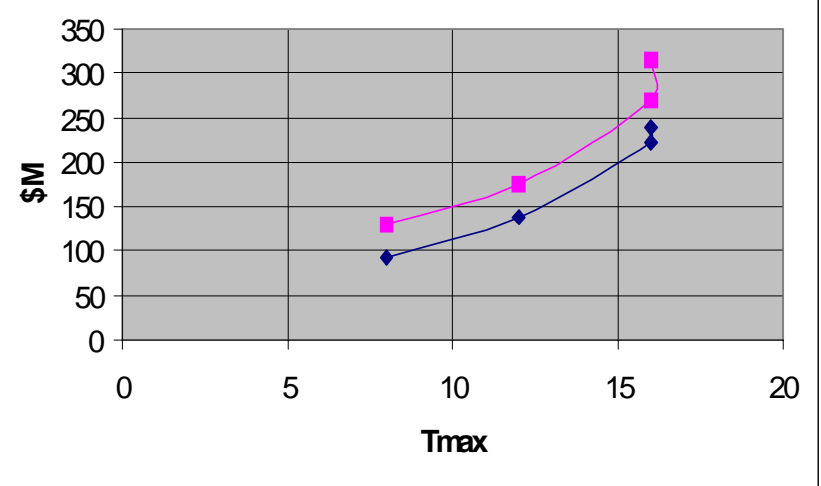

Figure 1. Tmax vs. Construction Cost. The scaled costs for the model machines are shown for stage 1 (lower curve) and stages 1 and 2 combined (upper curve). The points on the smooth curves are for the $40 \pi$ models at 8 , 12 , and $16 \mathrm{GeV}$ with circumference $0.75,1$, and 1.5 times $\mathrm{C}_{\mathrm{B}}$, respectively. The extra points at $16 \mathrm{GeV}$ correspond to the baseline $60 \pi$ Proton Driver[1]. The stage 2 cost for the bPD is higher than the $16 \mathrm{GeV}$ model because of its larger acceptance. The stage 1 cost for the bPD is shown at $16 \mathrm{GeV}$, though its magnet power supplies and reused Booster $53 \mathrm{MHz}$ rf system limit it to $12 \mathrm{GeV}$. All models have the same calculated Laslett tune shift as the bPD. Stage 2 models provide $1 \mathrm{MW}$ beam power. Stage 1 models inject $1.2 \times 10^{14}$ into the MI, except the $8 \mathrm{GeV}$ case, which provides $1.6 \times 10^{14}$.

\section{REFERENCES}

[1] W. Chou, C. Ankenbrandt, and E. Malamud, Eds., "The Proton Driver Design Study", Fermilab-TM-2136 (Dec., 2000). http://www-bd.fnal.gov/pdriver/reports.html [2] http://www-bd.fnal.gov/pdriver/designreport/appb.pdf

*This work was supported in part by grants from the Illinois Board of Higher Education, the Illinois Department of Commerce and Community Affairs and the U.S. Department of Energy. 